This article was downloaded by: [Bhatnagar-Mathur, Pooja]

On: 6 December 2009

Access details: Access Details: [subscription number 917443629]

Publisher Taylor \& Francis

Informa Ltd Registered in England and Wales Registered Number: 1072954 Registered office: Mortimer House, 3741 Mortimer Street, London W1T 3JH, UK

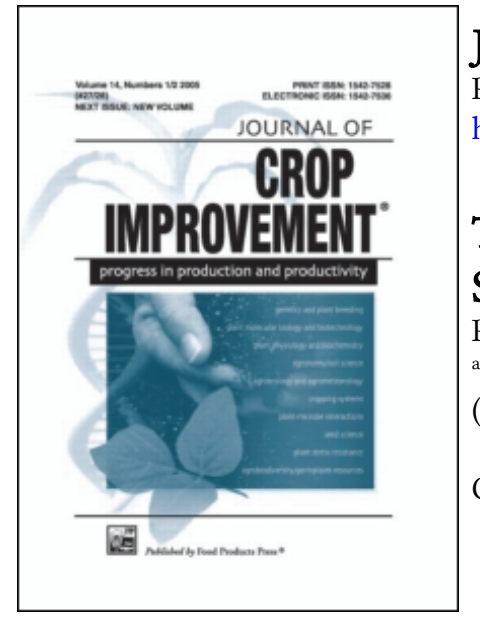

\title{
Journal of Crop Improvement
}

Publication details, including instructions for authors and subscription information:

http://www.informaworld.com/smpp/title $\sim$ content=t792303981

\section{Transgenic Strategies for Improved Drought Tolerance in Legumes of} Semi-Arid Tropics

Pooja Bhatnagar-Mathur ${ }^{\text {a }}$;. Shridhar Rao ${ }^{\text {; }}$ Vincent Vadez ${ }^{\text {a }}$; Kiran K. Sharma ${ }^{\text {a }}$

${ }^{a}$ Genetic Transformation Laboratory, International Crops Research Institute for the Semi-Arid Tropics (ICRISAT), Patancheru, Andhra Pradesh, India

Online publication date: 04 December 2009

To cite this Article Bhatnagar-Mathur, Pooja, Rao, J. Shridhar, Vadez, Vincent and Sharma, Kiran K.(2009) 'Transgenic Strategies for Improved Drought Tolerance in Legumes of Semi-Arid Tropics', Journal of Crop Improvement, 24: 1, 92 111

To link to this Article: DOI: $10.1080 / 15427520903337095$

URL: http://dx.doi.org/10.1080/15427520903337095

\section{PLEASE SCROLL DOWN FOR ARTICLE}

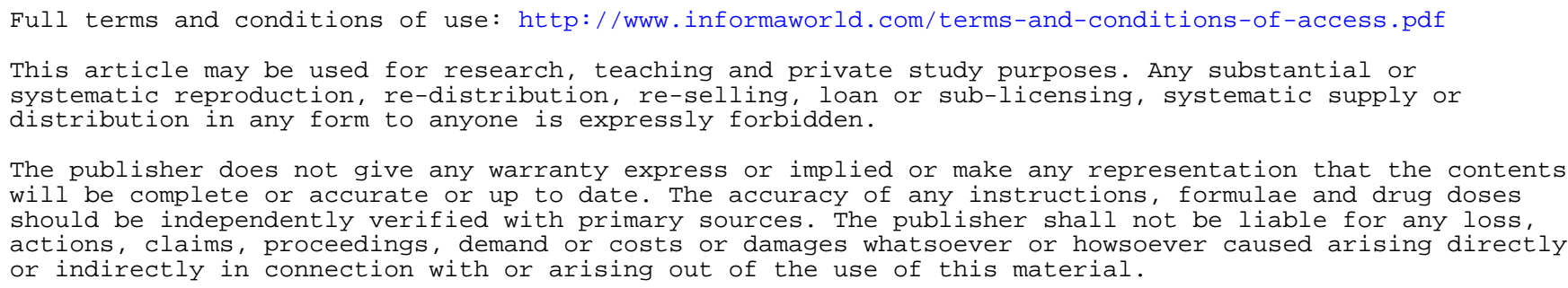




\title{
Transgenic Strategies for Improved Drought Tolerance in Legumes of Semi-Arid Tropics
}

\author{
POOJA BHATNAGAR-MATHUR, J. SHRIDHAR RAO, \\ VINCENT VADEZ, and KIRAN K. SHARMA \\ Genetic Transformation Laboratory, International Crops Research Institute \\ for the Semi-Arid Tropics (ICRISAT), Patancheru, Andhra Pradesh, India
}

\begin{abstract}
Water deficit is the most prominent abiotic stress that severely limits crop yields, thereby reducing opportunities to improve livelihoods of poor farmers in the semi-arid tropics (SAT) where most of the legumes, including groundnut and chickpea, are grown. Sustained long-term efforts in developing these legume crops with better drought tolerance through conventional breeding have been met with only limited success mainly because of an insufficient understanding of the underlying physiological mechanisms and lack of sufficient polymorphism for drought tolerance-related traits. Exhaustive efforts are being made at the International Crop Research Institute for Semi-Arid Tropics (ICRISAT) to improve crop productivity of the SAT crops by comprehensively addressing the constraints caused by water limitations. The transgenic approach has been used to speed up the process of molecular introgression of putatively beneficial genes for rapidly developing stress-tolerant legumes. Nevertheless, the task of generating transgenic cultivars requires success in the transformation process and proper incorporation of stress tolerance into plants. Hence, evaluation of the transgenic plants under stress conditions and understanding the physiological effect of the inserted genes at the whole plant level is critical. This review focuses on the recent progress achieved in using transgenic technology to improve drought tolerance, which includes evaluation of drought-stress response and protocols developed for testing transgenic plants under near-field conditions. A trait-based approach was considered, in which yield was dissected into components.
\end{abstract}

Address correspondence to Pooja Bhatnagar-Mathur at Genetic Transformation Laboratory, International Crops Research Institute for the Semi-Arid Tropics (ICRISAT), Patancheru, Andhra Pradesh 502 324, India. E-mail: p.bhatnagar@cgiar.org 
Yield $(Y)$ is defined as transpiration (T) $x$ transpiration efficiency (TE) $x$ barvest index (HI).

KEYWORDS drought, transgenic, chickpea, groundnut, transpiration efficiency, water-use efficiency

\section{INTRODUCTION}

Climate change is a major global concern that can make dryland agriculture even more risk-prone, especially in the developing world. Abiotic stresses are a primary cause of crop loss worldwide, reducing average yields by $>50 \%$ in most major crops (Boyer 1982; Bray, Bailey-Serres, \& Weretilnyk 2000). Crops are often exposed to multiple stresses in many regions. Approximately, $19 \%$ of the world's agricultural land is subject to salt stress and 5\% to drought stress (FAO, 1996).

The semi-arid tropics (SAT) cover parts of 55 developing countries and account for about 1.4 billion people of the world. The SAT has very short growing seasons, separated by very hot and dry periods. In addition, rapid and unforeseen disturbances in these environments have resulted in stressful conditions, which include paucity of water for long periods because of lack of irrigation, infrequent rains, or lowering of water table. These conditions cause drought stress. On the other hand, excess water from rain, cyclones, or frequent irrigation results in flooding, submergence or anaerobic stress. Climatologists believe that the changing global climate might produce even more severe and widespread dry conditions in these regions, with potentially serious consequences for agriculture and food availability (Wenzel \& Wayne 2008). In addition, water limitation may prove to be a critical constraint to crop productivity under future scenarios (Fischer et al. 2001). Since rainfed agriculture, particularly in the developing countries of the SAT, contributes significantly to total food production, food security will be unsustainable in the absence of dramatic yield increases in the marginal environments, especially in the drought prone areas.

Conventional breeding and enhanced management practices have addressed several constraints that limit crop productivity or quality, but there are situations where the existing germplasm lacks the required traits. While plant breeding in the past has relied heavily on empirical approaches for drought tolerance in crop plants, there is a broad consensus that strategic approaches based on sound physiological and genetic understanding of yield will also be required if further yield gains are to be achieved (Jackson et al. 1996; Miflin 2000; Slafer 2003; Snape et al. 2001).

Broadly, the drought response of plants can be divided into three mechanisms: 1) drought escape, 2) drought avoidance, and 3) drought tolerance. To date, the most important contribution to drought tolerance has 
come from breeding for altered phonological traits like early flowering and maturity (Araus et al. 2002) that essentially involves escape from drought conditions rather then tolerance per se. Besides, plants undergo drought postponement either by conserving water or by enhanced water uptake, which is usually achieved by structural modifications such as increased cuticle thickness, deeper roots, reduced leaf area, or reduced stomatal conductance (Jones 2004). Limiting stomatal conductance can result in high water-use efficiency (WUE), which is a trait for postponement of stress. Under extreme stress, tolerance is about survival and not productivity. The drought tolerance is achieved through changes in the biochemical composition that protect the macromolecules and membranes or maintain cell turgor through osmotic adjustment.

Yield losses due to constraints like drought are highly variable in nature depending on the stress timing, intensity, and duration, which is related to location-specific environmental stress factors such as high irradiance and temperature that make breeding for drought tolerance through conventional approaches difficult. Since most crop plants have not been selected for meeting exigencies caused by these stress factors, their capacity to adjust to such conditions is usually limited. Nevertheless, efforts to breed crop species for high WUE and stomatal conductance have met with limited success. This is in part because the physiological data and information about the molecular events underlying the abiotic stress responses are scarce, besides the non-availability of molecular tools that could help in assisting the breeding activities for such complex traits. Besides, a main constraint in the advancement efforts of crop improvement for drought tolerance includes insufficient know-how of tolerance mechanisms, a poor understanding of its low inheritance, and a scarcity of efficient techniques for screening of germplasm and breeding material.

\section{TRANSGENIC INTERVENTIONS FOR DROUGHT TOLERANCE}

Nevertheless, agricultural biotechnology has the potential to address this challenge and can play a role in developing crops that use water more efficiently, thus reducing the negative consequences of drought. The use of genetic engineering technology potentially offers a more targeted gene-based approach for improving plant's adaptation to water-limiting conditions. Transgenic approaches offer a powerful means of gaining valuable information to understand the mechanisms governing stress tolerance, providing a complementary means for the genetic betterment of the genome of field crops and thus promising the alleviation of some of the major constraints to crop productivity in the developing countries (Sharma \& Ortiz 2000). When a plant is subjected to abiotic stress, a number of genes are turned on, resulting in increased levels of several osmolytes and proteins, some of which may 
be responsible for conferring a certain degree of protection from these stresses. Therefore, it will likely be necessary to transfer several potentially useful genes into the same plant in order to obtain a high degree of tolerance to drought or salt stress. Novel genes accessed from exotic sourcesplants, animals, bacteria, even viruses_can be introduced into the crop through biolistics or by using Agrobacterium-mediated genetic transformation (Sharma \& Kavanya 2002). Further, it is possible to control the timing, tissue-specificity, and expression level of transferred genes for their optimal function.

Association of several traits with tolerance has been tested in transgenic plants. The results of transgenic modifications of biosynthetic and metabolic pathways indicate that higher stress tolerance can be achieved by engineering. However, the results of simulation modeling also suggest that changes in a given metabolic process, relatively apart from the yield architecture, may end up with little benefit for actual yield under stress (Sinclair, Purcell, \& Sneller 2004). Various transgenic technologies have been used to improve stress tolerance in plants (Allen 1995). In recent years, the genes responsible for low-molecular-weight metabolites have been shown to confer increased tolerance to salt or drought stress in transgenic dicot plants (mainly tobacco). Metabolic traits, especially pathways with few enzymes, have been genetically characterized and are more amenable to manipulations than structural and developmental traits. Genetically engineered plants for single-gene products include those encoding for enzymes required for the biosynthesis of osmoprotectants (Tarczynski, Jensen, \& Bohnert 1993; Kavikishore et al. 1995; Hayashi et al. 1997), or modifying membrane lipids (Kodama et al., 1994; Ishizaki-Nishizawa et al., 1996), late embryogenesis proteins (Xu et al., 1996), and detoxifying enzymes (McKersie et al., 1996). However, the results of the transfer of a single trait driven by a single protein are unlikely to improve a plant's tolerance beyond the short-term effects that have been reported. Hence, multiple mechanisms to engineer water stress tolerance must be utilized (Bohnert, Nelson, \& Jenson 1995). Indeed, drought being an extremely complex phenomenon controlled by multiple genes and regulatory pathways, drought tolerance has proved much more difficult to engineer into plants than more simply inherited traits governed by single genes. Therefore, many genes involved in stress response can be simultaneously regulated by using a single gene encoding stress-inducible transcription factor (Kasuga et al. 1999), thereby offering the possibility of enhancing tolerance towards multiple stresses including drought, salinity, and freezing. Transcription factors modulate the expression of a cascade of stress-inducible genes to impart tolerance to stressed plants (Bartels \& Sunkar 2005; Chinnusamy, Jagendorf, \& Zhu 2005).

Several recent reviews have provided comprehensive lists of genes shown to confer drought tolerance in plants (Wang, Vinocur, \& Altman 2003; Zhang et al. 2004; Umezawa et al. 2006) that support the prospects of 
genetically engineering for drought tolerant crops. However, in most of the cases, the evidence indicating that these genes confer drought tolerance is based on laboratory-grown plants under artificial drought conditions. The translation of drought tolerance from research experiments with model species in the laboratory to crop species in the field depends on the ability to transfer the mechanism of tolerance and, on whether the mechanism of tolerance will actually lead to increased biomass or yield under drought stress in crop species. Again, here it is important to identify and understand the kind of drought tolerance that is the target. For example, genes that confer desiccation tolerance in the laboratory may not be useful for enhancing yield in the field, although they are the object of extensive studies. In order to improve the genetic make-up of the agronomically important crop plants for their integration into the breeding programs, the research focus should be on crop productivity where their successful deployment in the field will require more detailed physiological studies than are typically reported (Chaves \& Oliveira, 2004).

\section{TRANSGENIC RESEARCH FOR DROUGHT TOLERANCE AT ICRISAT}

Legumes such as chickpea and groundnut constituting the important food and oilseed crops of the SAT are mostly grown in low-input, rain-fed agriculture of the world and suffer from drought due to insufficient, untimely, and erratic rainfall in these climates that become major constraints to crop productivity. Globally, on an average, about 20\% of the land surface is under drought at any one time, and the proportion of the land surface in extreme drought is predicted to increase from 1\% for the present day to 30\% by the end of the $21^{\text {st }}$ century (Burke, Brown, \& Christidis 2006). Thus, drought represents the major constraint to increase their yield, and drought tolerance therefore is a major aim of breeding of these legume crops. Exhaustive efforts are being made at the International Crop Research Institute for Semi-Arid Tropics (ICRISAT) to improve the crop productivity of the SAT crops by comprehensively addressing the constraints due to water limitations. This is done through cutting-edge, knowledge-based breeding practices complemented adequately by genomics and genetic transformation technologies that could lead to simpler and more effective gene-based approach for improving drought tolerance.

There has been a general consensus that increased crop performance of the legumes under drought conditions can be achieved through improvements in total water use, WUE, and harvest index (Nigam et al. 2005; Vadez et al. 2008). Amongst these, transpiration efficiency (TE), one of the components of WUE, is very critical to plant performance under water-limiting conditions, and hence new breakthroughs in water-saving strategies could be attained by improving WUE in these crop species. Sustained long-term 
efforts in developing groundnut and chickpea crops with better drought tolerance/WUE through conventional breeding so far have had only limited success. This is mainly due to an insufficient understanding of the physiological mechanisms underlying WUE and a lack of sufficient polymorphism for these traits. As a consequence, there is difficulty in discovering molecular markers for WUE that have the potential to ease the breeding process. Drought tolerance seems to be controlled by complex sets of traits that may have evolved as separate mechanisms in different groups of plants, thereby making the breeding of these using conventional methods difficult. Therefore, a continuing need has been felt to integrate biotechnological approaches with plant physiology and plant breeding for an efficient application of these tools for crop improvement. Hence, efforts are underway to use genetic engineering approaches for speeding up the process of improving resilience in these legumes to drought stress by specifically targeting and more quickly inserting genes known to be involved in plant response to stress.

At ICRISAT, research is being carried out to impart drought tolerance using both osmoregulatory and regulatory gene approaches. Because it is believed that osmoregulation is one of the best strategies for abiotic stress tolerance, especially if osmoregulatory genes could be triggered in response to drought, salinity, and high temperature. A widely adopted strategy has been to engineer certain osmolytes or cause overexpression of such osmolytes in plants to breed stress-tolerant crops (Ishitani et al. 1997; Holmstrom et al. 2000; Delauney \& Verma 1993; Nanjo et al. 1999; Zhu et al. 1998; Yamada et al. 2005). We have introduced an osmoregulatory gene, P5CSF129A, encoding the mutagenized D1-pyrroline-5-carboxylate synthetase (P5CS) for the overproduction of proline in chickpea. The hypothesis for the present study was that the introduction of the P5CSF129A gene into chickpea might result in the accumulation of elevated amounts of endogenous proline, and consequently improve plant production into this important pulse crop. The transgenic chickpea plants were extensively evaluated under greenhouse conditions for various physiological, molecular, and biochemical characters under a typical dry-down setup for water deficits. The accumulation of proline in several transgenic events was more pronounced and increased significantly in the leaves when exposed to water stress coupled by a decrease in the free radicals as measured by a decrease in the malonaldehyde (MDA) levels, a lipid peroxidation product. Eleven transgenic events that accumulated high proline (2-6 folds) were further evaluated in the greenhouse experiments based on their TE, photosynthetic activity, stomatal conductance, and root length under water stress. Almost all the transgenic events showed a decline in transpiration at lower values of the fraction of transpirable soil water (TSWV; dryer soil), and extracted more water than their untransformed parents. However, the overexpression of proline appeared to have no beneficial effect on the biomass accumulation since only a few events showed a significant increase in the biomass production toward the 
end of the progressive drying period. In any case, the overexpression of P5CSF129A gene resulted only in a modest increase in TE, thereby indicating that the enhanced proline had little bearing on the components of yield architecture, which are significant in overcoming the negative effects of drought stress in chickpea. These results agree with the previous reports in other crops (Turner \& Jones 1980; Morgan 1984; Serraj \& Sinclair 2002; Turner et al. 2007) and, in our above assessment, the gene affecting single protein might be less efficient in coping with water-limiting conditions.

Hence, to cater to the multigenicity of the plant response to stress, a strategy to target transcription factors that regulate the expression of several genes related to abiotic stress was considered. The purpose was to develop a large number of groundnut and chickpea transgenic plants carrying the DREB1A transcription factor from Arabidopsis, driven by a stress-inducible promoter from $r d 29 A$ gene from A. thaliana. Regulatory genes or transcription factors, more specifically those belonging to the AP2/ERF family, have previously been shown to improve stress tolerance under lab conditions by regulating the coordinated expression of several stress-related genes in heterologous transgenic plants (Kasuga et al. 1999, 2004; Behnam et al. 2006; Bhatnagar-Mathur et al. 2007). Since, improving WUE of a plant is a complex issue, and efforts to breed groundnut genotypes for high TE and stomatal conductance have obtained limited success, regulatory genes or transcription factors have a potential to improve stress tolerance by regulating the coordinated expression of several stress-related genes in heterologous transgenic plants. Plants overexpressing the P5CSF129A and DREB1A genes demonstrated substantial increase in TE under experimental greenhouse conditions (Bhatnagar-Mathur et al. 2007, 2009a). A few transgenic events with contrasting responses have been selected for further detailed studies on the gas exchange characteristics of leaves. Besides, the biochemical responses of plants under identical conditions of water stress have been examined critically for further understanding of the mechanisms underlying environmental stress resistance in these transgenic events (Bhatnagar-Mathur et al. 2009b).

\section{Choosing a Right Promoter is Important}

Tissue specificity of transgene expression is also an important consideration while deciding on the choice of the promoter to increase the level of expression of the transgene for any transgenic technology. Thus, the strength of the promoter and the possibility of using stress-inducible, developmental-stage or tissue-specific promoters need to be considered (Bajaj et al. 1999). While for some gene products, such as LEA3, which are needed in large amounts, a very strong promoter is needed, for others like enzymes for polyamine biosynthesis, it may be better to use an inducible promoter of moderate strength. So far, most of the promoters that have been 
most commonly used in the development of abiotic stress tolerant plants have been constitutive in nature. These include the CaMV 35S, ubiquitin 1, and actin promoters, which by and large express the downstream transgenes in all organs and at all the stages of growth and development. However, constitutive overproduction of molecules, such as trehalose (Romero et al. 1997) or polyamines (Capell et al. 1998), causes abnormalities in plants grown under normal conditions. In addition, since the production of these molecules can be metabolically expensive, the use of a stress-inducible promoter may be desirable. Various types of abiotic stresses induce a large number of well-characterized and useful promoters in plants. The transcriptional regulatory regions of the drought-induced and cold-induced genes have been analyzed to identify several cis-acting and trans-acting elements involved in abiotic stress induced gene expression (Shinwari 1999). For complex traits like drought, an ideal inducible promoter should be the one that does not show any basal level of gene expression in the absence of inducing agents, besides resulting in an expression that is reversible and dose-dependent. Most of the stress-inducible promoters contain an array of stress-specific cis-acting elements that are recognized by the requisite transcription factors. The A. thaliana $r d 29 A$ and $r d 29 B$ are stress responsive genes differentially induced under abiotic stress conditions. While the rd29A promoter includes both DRE and ABRE elements and dehydration, high salinity and low temperatures induce the gene; the $r d 29 B$ promoter induces only ABREs; and the induction is ABA-dependent. Overexpression of $D R E B 1 A$ transcription factors gene under the control of stress-inducible promoter $r d 29 A$ has been reported to show a better phenotypic growth of the transgenic plants than the ones obtained using the constitutive CaMV35S promoter (Kasuga et al. 1999; Bhatnagar-Mathur et al. 2007). Gene expression is induced by the binding of DREB1A, which is itself induced by cold and water stress, to cis-acting DRE elements in the promoters of genes such as $r d 29 A, r d 17, \operatorname{cor} 6.6, \operatorname{cor} 15 A$, erd10, and kin1, initiating synthesis of gene products imparting tolerance to low temperatures and water stress in plant. These basic findings on stress promoters have led to a major shift in the paradigm for genetically engineering stress tolerant crops (Katiyar-Aggarwal, Agarwal, \& Grover 1999). A thorough understanding of the underlying physiological processes in response to different abiotic stresses can efficiently/ successfully drive the choice of a given promoter or transcription factor to be used for transformation.

\section{DROUGHT EVALUATION OF TRANSGENICS: A REVIEW}

The task of generating transgenic cultivars is not only limited to the success in the transformation process, but also proper evaluation of the stress tolerance. Understanding the physiological effect of the inserted genes at the whole-plant 
level remains a major challenge. Most of the procedures that have been followed to impose stress for the phenotypic evaluation of transgenic plants for their response to drought and other stresses have been questioned (Sinclair, Purcell, \& Sneller 2004; Bhatnagar-Mathur, Vadez, \& Sharma 2008). A large number of studies have been carried out to evaluate different transgenic constructs in different plant species to drought stress. Although, there has been a tendency to report in detail the expression of the transgenes as well as the level of metabolite increase due to the transgene, fewer details are given with regard to the method used to evaluate the stress response.

This lack of detail applies mostly to drought stress where a variety of methods have been applied in various laboratory settings, which include 1) osmotically adjusted media, 2) detaching leaves, and 3) withholding water from soil-grown plants. Also, the protocols used for the evaluation of transgenic plants for abiotic stresses often involved the use of young plants grown in small pots, disregarding water content in pots, usually maintained under inappropriate light and growth conditions (Tarczynski, Jensen, \& Bohnert 1993; Pilon-Smits et al. 1996; Xu et al. 1996; Pellegrineschi et al. 2004). Stress conditions used to evaluate the transgenic material in most of the reports so far have been too sharp (Shinwari et al. 1998), (Nanjo et al. 1999; Garg et al. 2002), meaning the plants are very unlikely to undergo them in a real field condition situation.

Most of the reported assays for drought tolerance typically involved the complete withholding of water until control plants wilted followed by re-watering and recovery (see Fujita et. al. 2005; Sakuma et. al 2006) where the measure of drought tolerance was often given as percent survival. Since slight differences in the degree of wilting can translate into large differences in survival, these tend to amplify the apparent tolerance. However, this approach is useful for assessing tolerance for extremely dry conditions that may be relevant for plant survival, but less relevant for crop productivity under field conditions. These protocols are in fact used to assess transgene response and expression, which unfortunately often becomes assimilated to "tolerance." Also, the experimental means of evaluation can be misleading, resulting in inconsistent data output that leads to conclusions far from convincing and meaningful (Pilon-Smits et al. 1995, 1996, 1999; Pardo, Reddy, \& Yang 1998; Sivamani et al. 2000; Sun et al. 2001; Lee et al. 2003; Pellegrineschi et al. 2004).

\section{Adequate Protocols for Transgenic Evaluation for Drought}

Unlike what seems to be a common practice in transgenic evaluation, applying drought does not consist simply in withholding water. One of the major limitations in the evaluation and screening of drought-tolerant genotypes/ transgenics has been the lack of a clear definition of the targeted drought. The lack of means to compare drought conditions across experiments, 
along with the differences in the timing, intensity, and duration of the moisture deficit conditions, makes the comparison of work by different groups relatively difficult. Indeed, we cannot investigate drought response of plants without understanding the different phases that a plant undergoes under drought in natural conditions. These steps have been described earlier (Ritchie 1982; Sinclair \& Ludlow 1986). Briefly, in phase I, water is abundant and the plant can take up all the water required for transpiration and stomata are fully open. During that stage, the water loss is mostly determined by the environmental conditions to which the leaves are exposed. During phase II, the roots are no longer able to supply sufficient water to the shoot and stomata progressively close to adjust the water loss to the water supply, so that leaf turgor is maintained. During phase III the roots are no longer able to supply sufficient water to the shoot, and stomata progressively close to adjust to the water loss to the water supply, so that leaf turgor is maintained.

At ICRISAT, we are developing a thorough understanding of the different phases that the plant undergoes under drought, which includes a more realistic physiological response to progressive soil drying. A proper control of soil moisture depletion is done to ensure that transgenic plants are exposed to stress levels and kinetics of water deficits approaching those occurring under field conditions. This involves designing dry-down experiments, where the response of plants to drought is taken as a function of the fraction of soil-water moisture available to plant (FTSW), which allows a precise comparison of stress imposed across experiments. Here, we base our index of stress intensity on FTSW, which represents the volumetric soil water available. Based on the transpiration, the plants can be partially compensated for the water loss to apply a milder stress condition which allows plants of different sizes to be exposed to similar stress levels. This protocol has the advantage of mimicking the situation a plant would face in the field, i.e., a progressive soil drying. This concept has been successfully adapted to assess the response of transgenic plants of groundnut and chickpea with $A t$ rd29A-driven DREB1A transgene as well as with the constitutively expressed P5CSF129A gene under contained greenhouse conditions (Bhatnagar-Mathur et al. 2004).

Briefly, in the pot studies under contained greenhouse conditions, the pots are filled with soil taken from the field and plants are initially grown under well-watered conditions with the air temperature regulated at approximately $22 \pm 2{ }^{\circ} \mathrm{C}$ before initiating the experimental treatments. In all the experiments, the plants are grown under well-watered conditions until flowering prior to imposing the drought treatment. In each experiment, the pots are divided into three subsets, each set having six replicates of plants. The pretreatment set is harvested at the beginning of the experiment to record the initial plant biomass. The other two sets are used as well-watered control and stress treatment and are harvested at end of the experiment. The afternoon before the stress treatment, all pots are fully watered (saturation) 
and allowed to drain overnight. Next morning, the pots are covered with white plastic bags around the stem to prevent direct soil evaporation. A small tube is inserted in plastic bags to re-water the pots during the experiment. The pots are weighed thereafter, and the weight serves as the initial target pots weight. Subsequently, the pots are regularly weighed every morning at a fixed time. The daily transpiration is calculated as the pot weight difference between two successive days. To control the rapid soil drying in the drought stress treatment and to allow plants to develop stress progressively and uniformly, drought-stressed plants are allowed to lose no more than $70 \mathrm{~g}$ of water daily. A partial re-watering of the stressed pots is done every morning after weighing to maintain a daily net water loss of $70 \mathrm{~g}$. In both the experiments, well-watered control plants of each genotype are maintained at initial target weight by adding the daily water loss back to the pots.

The above experiment is terminated when the daily transpiration rate of drought-stressed plants decreases to less than 10\% that of the wellwatered plants. At this endpoint, soil water is no longer available to meet the transpiration demand (Sinclair \& Ludlow 1986). The transpiration data are analyzed by the procedure previously described by Ray and Sinclair (1997) and Sinclair and Ludlow (1986). To minimize the influence of large variations in daily transpiration across days, the daily transpiration rates $(\mathrm{T})$ of the drought-stressed pots are normalized against the transpiration rate of the well-watered plants each day. Transpiration ratios (TR) are calculated by dividing daily transpiration of each individual plant in the drought-stressed regime by the daily mean transpiration of the well-watered control plants for each genotype. The values of TR vary among individual plants because of plant size differences among plants within the treatments. To facilitate the comparisons among plants, a second normalization (normalized transpiration ratio, NTR) is done such that the TR of each plant is divided by the TR of that plant averaged across the first three days of the drying cycle, i.e., before any water stress starts affecting $T$. The transpirable soil water available to the plant in each pot is calculated as the difference between the initial pot weight (field capacity) and the endpoint (no more transpirable water). The use of transpirable soil water as the basis of comparing plant response to soil drying under a range of conditions has been effectively used in a number of studies (Ray \& Sinclair 1997; Serraj, Purcell, \& Sinclair 1999).

Sensitivity of the plant to the amount of available water in the soil profile under water deficit is used as a criterion for comparing drought intensity across experiments (Ritchie 1982). The value of FTSW threshold is calculated from the difference between the water content of the profile after a thorough wetting to saturation and the water content of the profile after healthy plants have exhausted the entire possible water source (transpirable soil water). This approach has been successfully used to monitor the response of different physiological processes to water deficit in many plant species 
(Sinclair \& Ludlow 1986; Ray \& Sinclair 1997, 1998; Serraj, Purcell, \& Sinclair 1999).

In our studies, the transgenic events of groundnut and chickpea were evaluated using the dry-down methodology, where a diversity of stress response patterns was observed, especially with respect to the NTR-FTSW relationship. The soil-moisture threshold where the transpiration rate begins to decline relative to control well-watered (WW) plants and the number of days needed to deplete the soil water was used to rank the genotypes using the average linkage cluster analysis. Five diverse events were selected from the different clusters for further testing. All the selected transgenic events were able to maintain a transpiration rate equivalent to the WW control in soils dry enough to reduce transpiration rate in the untransformed controls. Various transgenic events in both the crops exhibited increased TE, which is an important component of plant performance under limited moisture conditions (Passioura, 1977). The TE, which is an important characteristic for plant breeding as a means of improving farm productivity under drought stress, is calculated as the ratio of biomass increase between initial and final harvest, divided by the total water transpired during that period. The most striking finding with the groundnut transgenic events was that some events showed up to $40 \%$ higher TE over the untransformed parents under drought stress. These differences can be considered very large when compared with the range of variation usually found for TE between germplasm accessions of peanut (Sheshshayee et al. 2006) and in many other crops (Krishnamurthy et al. 2007) where only a 20-30\% difference in TE was observed across a mapping population developed between high and low TE parents.

\section{Roots Are Also Critical For Evaluations}

Much has been said about the potential of roots to improve crop yield and resilience under drought. Yet, very little breeding, specifically for root traits, has been achieved in groundnut. Although, some progress has been achieved, there are bottlenecks in assessing roots in a large number of genotypes that can be meaningful for plant breeders. This might be partly because of the time-consuming methods to measure rooting differences, limiting their use in breeding (Kashiwagi et al. 2006). Water uptake is probably crucial during key stages of plant growth and development like flowering and grain filling (Boyer \& Westgate 1984), and small differences in water uptake at these stages can bring large yield benefits (Boote et al. 1982). However, so far most of the studies being carried out by various groups on root measurements do not address these critical parameters. As we progress in the direction of understanding the role of roots using a unique lysimetric system (described below), we advocate that more focus should be put on the functionality of roots rather than on their morphology, 
in particular with regards to direct water-uptake measurements and their related kinetics (Vadez et al. 2008).

In our transgenic events of groundnut and chickpea, DREB1A certainly appeared to confer a drought-avoidance mechanism by improving WUE. However, it was worthwhile testing whether DREB1A could also induce drought avoidance through better water capture. It is well known that under water stress, plants tend to increase their root/shoot ratio. We tested whether DREB1A gene could have an effect on root growth under water deficit. Interestingly, we found that DREB1A clearly induced a root response under water deficit conditions (Vadez et al. 2008). This response enhanced root growth under water deficit, in particular in the deep soil layers. Consequently, water uptake under water deficit was enhanced up to 20\%-30\% in some transgenic events compared with the WT and was well related $\left(\mathrm{r}^{2}=0.91\right)$ with the root dry weight below the $40 \mathrm{~cm}$ soil depth (Vadez et al. 2007). This was an interesting finding where DREB1A transcription factor certainly had an impressive effect on the root growth under water deficit. It was expected though, as DREB1A appears to be a major "switch" for a cascade of genes that are activated under water deficit. These results supported our argument that the capacity of roots to take up water should also be considered in the context of the capacity of shoots to limit their water loss in a comprehensive manner (Vadez et al. 2008).

\section{THE LYSIMETRIC SYSTEM}

At ICRISAT, we have designed and tested a lysimetric system in which plants are grown in long and large polyvinyl chloride (PVC) cylinders (20 $\mathrm{cm}$ in diameter and $1.2 \mathrm{~m}$ long) that can be tested either under contained greenhouse conditions or even for conducting strip trials for the selection of transgenic events (Vadez et al. 2008). Here, utmost attention is paid to develop a system that mimics the field conditions as closely as possible. The soil volume available to each individual plant is almost equivalent to the soil volume available in the field at usual planting densities $\left(25-30\right.$ plant $\left.\mathrm{m}^{-2}\right)$. This system allows measurement of water uptake by plants grown in a real soil profile under conditions of water deficit and to understand how roots contribute to drought tolerance and stress conditions. The soil packing is done using soil sieved in particles smaller than $1 \mathrm{~cm}$. This allows controlling the bulk density to be approximately 1.4, which is the standard value for Alfisols. To ensure that moisture is available in all parts of the cylinders, $40 \mathrm{~kg}$ of dry soil is initially filled in each cylinder, keeping the soil level similar in each tube. A prior assessment of the water needed to fill the profile before saturation point is determined such that the water-holding capacity of the soil is approximately $20 \%$. Therefore, $8 \mathrm{~L}$ of water is added to the first $40 \mathrm{~kg}$ of soil. An additional $10 \mathrm{~kg}$ of dry soil is added to each cylinder soon after 
the water has penetrated the profile and again $2 \mathrm{~L}$ water is added. All the cylinders have a very similar bulk density, close to 1.4 .

At planting, the soil is wetted and seeds planted at a rate of 2 /cylinders that are later thinned to 1 plant per cylinder where irrigation is done at regular intervals. This system is most suited to tailoring a number of drought regimes for a range of crops. In our preliminary experiments, we have imposed the last irrigation (to saturate the soil profile) at about one week after flowering. At that stage, low-density polyethylene beads are applied to all cylinders ( $600 \mathrm{~mL}$ per cylinder to have a bead layer of approximately $2 \mathrm{~cm}$ ). The purpose of the beads is to limit soil evaporation by $90 \%$ (Vadez et al. 2008). Therefore, the regular weighing of the cylinders provides transpiration data with beads allowing peg penetration in the case of groundnut (Vadez et al. 2008). As this system simulates the natural soil profile of the field, it allows the penetration and growth of the roots all along the length of the tube. At the end of the experiment, the tubes can be washed for easy extraction of intact roots for further analysis through scanning for root surface area, root-length density, and root volume at varying depths that are crucial parameters to screen the genotypes for differences in root traits, correlating their water-uptake performance in general. The phenotypic evaluation of the transgenic events of groundnut and chickpea using the lysimetric system clearly indicated that the stress-inducible expression of DREB1A appeared to confer capture capacity in several transgenic events when compared with their untransformed parents. Moreover, several transgenic events appeared to have consistently higher TE than the WT across different water regimes. Although, these results are very encouraging, one should be cautiously certain about the fact that improved TE in these lines is not at the cost of other yield architecture components.

\section{YIELD IS THE LITMUS TEST}

ICRISAT has been involved for many years in the development of groundnut breeding lines having high WUE. These have been assessed via a major component of drought tolerance, viz., evapotranspiration efficiency (TE), because WUE has been identified as a major contributor to pod yield under intermittent water-deficit conditions (Wright et al. 1993; Wright, Nageswara Rao, \& Farquhar 1994). However, a yield-based approach as selection criteria to select genotypes where the lines are selected based on yield has proven to be more successful under water deficit (Nigam et al. 2005). This means that each component of the yield architecture cannot be addressed independently of the other. Ultimately, all efforts are made to study the component traits to comprehend their contribution to the yield in general according to the Passioura's model $\mathrm{Y}=\mathrm{T}^{*} \mathrm{TE} * \mathrm{HI}$ (Passioura 1977). Following this, preliminary yield trials of the groundnut transgenic events have been 
conducted both under contained greenhouse and field and/or in controlled lysimetric conditions, where the effect of drought on the various potential component traits of yield architecture (Passioura 1977), such as T, TE, or harvest index (HI), were addressed comprehensively under terminal and intermittent drought conditions. Here, under terminal drought, irrigation was completely suppressed at 40 days after sowing; under intermittent drought stress, re-watering $(1 \mathrm{~L})$ was done at 6, 9, and 12 weeks after stress imposition. The results obtained so far have been encouraging. They indicated significantly higher yields in a few selected transgenic events when compared with their untransformed parent under drought (unpublished data). Efforts are ongoing for reconfirmation of these observations under drought stress in these transgenic events before dissecting the individual component traits of yield.

\section{CONCLUSIONS}

A very efficient delivery system is available at ICRISAT for the assessment of drought tolerance and yield in legumes, such as chickpeas and groundnuts, which would immensely influence the progress in the transgenic research for drought tolerance in the future. Results obtained with these legumes provide evidence that transgenic interventions attempted to introgress genes putatively involved in drought tolerance has a potential to further improve drought tolerance and thereby increase and stabilize yield in the SAT regions. These results are very encouraging because the range of variation observed for TE was higher than what has been found with recombinant inbred lines (RIL) populations. Further, the fact that transgenic events showing such large phenotypic contrast, while being isogenic for one inserted gene, provides great opportunities to reinvestigate the mechanisms of drought tolerance in these legumes. This might in the near future lead to significant benefits in raising drought-tolerant genotypes. Further, the identification of other physiological traits linked to drought tolerance in our ongoing studies with these transgenic events is expected to provide more insights into the mechanisms of drought-stress tolerance both under greenhouse and field conditions.

\section{REFERENCES}

Allen, R.D. 1995. Dissection of oxidative stress tolerance using transgenic plants. Plant Physiol. 107: 1049-1054.

Araus, J.L., G.A. Slafer, M.P. Reynolds, and C. Royo, 2002. Plant breeding and water stress in C3 cereals: What to breed for? Ann. Bot. 89: 925-940.

Bajaj, S., J. Targolli, L.F. Liu, T.H. Ho, and R. Wu. 1999. Transgenic approaches to increase dehydration stress tolerance in plants. Mol. Breed. 5:493-503. 
Bartels, D., and R. Sunkar. 2005. Drought and salt tolerance in plants. Crit. Rev. Plant Sci. 21:1-36.

Behnam, B., A. Kikuchi, F. Celebi-Toprak, S. Yamanaka, M. Kasuga, K. YamaguchiShinozaki, and K.N. Watanabe.2006. The Arabidopsis DREB1A gene driven by the stress-inducible $r d 29 A$ promoter increases salt-stress tolerance in proportion to its copy number in tetrasomic tetraploid potato (Solanum tuberosum). Plant Biotech. 23:169-177.

Miflin, B.J. 2000. Crop biotechnology. Where now? Plant Physiol. 123:17-28

Bhatnagar-Mathur, P., M.J. Devi, R. Serraj, K. Yamaguchi-Shinozaki, V. Vadez, and K.K. Sharma. 2004. Evaluation of transgenic groundnut lines under water limited conditions. Int. Arch. Newsl. 24:33-34.

Bhatnagar-Mathur, P., M.J. Devi, V. Vadez, and K.K. Sharma. 2009a. Differential antioxidative responses in transgenic peanut bear no relationship to their superior transpiration efficiency under drought stress. J. Plant Physiol. 166: 1207-1217.

Bhatnagar-Mathur, P., M.J. Devi, V. Vadez, D.P.S. Verma, and K.K. Sharma. 2009b. Over expression of Vigna P5CSF129A gene in chickpea for enhancing drought tolerance. Mol. Breed. 23:591-606.

Bhatnagar-Mathur, P., D.S. Reddy, M. Lavanya, K. Yamaguchi-Shinozaki, and K.K. Sharma. 2007. Stress-inducible expression of Arabidopsis thaliana DREB1A in transgenic peanut (Arachis hypogaea L.) increases transpiration efficiency under water-limiting conditions. Plant Cell Rep. 26:2071-82.

Bhatnagar-Mathur, P., V. Vadez, and K.K. Sharma. 2008. Transgenic approaches for abiotic stress tolerance in plants: Retrospect and prospects. Plant Cell Rep. 27: 411-424.

Bohnert, H.J., D.F. Nelson, and R.G. Jenson. 1995. Adaptation to environmental stresses. Plant Cell 7:1099-1111.

Boote, K.J., J.R. Stansell, A.M. Schubert, and J.F. Stone. 1982. Irrigation, water use and water relations. In Peanut science and technology, eds. H.E. Patee, and L.T. Young, pp. 164-205. Yoakum, TX: American Peanut Research and Education Society.

Boyer, J.S. 1982. Plant productivity and environment. Science 218:443-448

Boyer, J.S., and M.E. Westgate. 2004. Grain yields with limited water. In Water-saving agriculture. Special issue, J. Exp. Bot. 55(407): 2385-2394.

Bray, E.A., J. Bailey-Serres, and E. Weretilnyk. 2000. Responses to abiotic stresses. In Biochemistry and molecular biology of plants, eds, W. Gruissem, B. Buchannan, R. Jones, pp. 1158-1249. Rockville, MD: American Society of Plant Physiologists.

Burke, E.J., S.J. Brown, and N. Christidis. 2006. Modeling the recent evolution of global drought and projections for the twenty-first century with the Hadley Centre climate model. J. Hydrometeorol. 7:1113-1125.

Capell, T., C. Escobar, H. Lui, H. Burtin, O. Lepri, and P. Christou.1998. Overexpression of the oat arginine decarboxylase cDNA in transgenic rice (Oryza sativa $\mathrm{L}$.) affects normal development patterns in vitro and results in putrescine accumulation in transgenic plants. Theor. Appl. Genet. 97:246-254.

Chaves, M.M., and M.M. Oliveira. 2004. Mechanisms underlying plant resilience to water deficits: prospects for water-saving agriculture. J. Exp. Bot. 55(407): 2365-2384. 
Chinnusamy, V., A. Jagendorf, and J.K. Zhu. 2005. Understanding and improving salt tolerance in plants. Crop Sci., 45:437-48.

Delauney A.J. and D.P.S. Verma.1993. Proline biosynthesis and osmoregulation in plants. Plant J. 4:215-223.

FAO. 1996. Quarterly Bulletin of Statistics. Rome: FAO

Fischer, G., M. Shah, H. van. Velthuizen, and F.O. Nachtergaele. 2001. Global agroecological assessment for agriculture in the $21^{\text {st }}$ century. Laxenburg, Austria: IIASA and FAO.

Fujita, Y., M. Fujita, R. Satoh, K. Maruyama, M.M. Parvez, M.Seki, K. Hiratsu, M. Ohme-Takagi, K.Shinozaki, and K. Yamaguchi-Shinozaki. 2005. AREB1 is a transcription activator of novel ABRE-dependent ABA signaling that enhances drought stress tolerance in Arabidopsis. Plant Cell 17: 3470-3488.

Garg, A.K., J.K. Kim, T.G. Owens, A.P. Ranwala, Y.C. Choi, L.V. Kochian, and R.J. Wu. 2002. Trehalose accumulation in rice plants confers high tolerance levels to different abiotic stresses. Proc. Natl. Acad. Sci. 99:15898-15903

Hayashi, H., Alia, L. Mustardy, P. Deshnium, M. Ida, and N. Murata. 1997. Transformation of Arabidopsis thaliana with the cod A gene for choline oxidase: accumulation of glycinebetaine and enhanced tolerance to salt and cold stress. The Plant J. 12: 133-142.

Hayashi, H., L. Mustardy, P. Deshnium, M. Ida, and N. Murata. 1997. Transformation of Arabidopsis thaliana with the codA gene for choline oxidase: accumulation of glycine betaine and enhanced tolerance to salt and cold stress. Plant J. 12:133-142.

Holmstrom K.O., S. Somersalo, A. Mandal, E.T. Palva and B. Welin. 2000. Improved tolerance to salinity and low temperature in transgenic tobacco producing glycine betaine. J Exp. Bot. 51:177-185.

Ishitani, M., L. Xiong, B. Stevenson, and J-K. Zhu. 1997. Genetic analysis of osmotic and cold stress signal transduction in Arabidopsis: Interactions and convergence of abscisic acid-dependent and abscisic acid-independent pathways. Plant Cell 9:1935-1949.

Ishizaki-Nishizawa, O., T. Fujii, M. Azuma, K. Sekiguchi, N. Murata, T. Ohtani, and T. Toguri. 1996. Low-temperature resistance of higher plants is significantly enhanced by a nonspecific cyanobacterial desaturase. Nat. Biotechnol. 14: 1003-1006.

Jackson, R.B., J. Canadell, J.R. Ehleringer, H.A. Mooney, O.E. Sala, and E.D. Schulze. 1996. A global analysis of root distributions for terrestrial biomes. Oecologia 108:389-411.

Jones, M.L. 2004. Changes in gene expression during senescence. In Plant cell death processes, ed. L. Nooden, pp. 51-72. San Diego, CA: Elsevier Science.

Kashiwagi, J., L. Krishnamurthy, R. Serraj, H.D. Upadhyaya, S.H. Krishna, S. Chandra, and V. Vadez. 2006. Genetic variability of drought-avoidance root traits in the mini-core germplasm collection of chickpea (Cicerarietinum L.). Euphytica 146:213-222.

Krishnamurthy, L., V. Vadez, M.J., Devi, R. Serraj, S.N. Nigam, M.S. Sheshshayee, S. Chandra and R. Aruna. 2007. Variation in transpiration efficiency and its related traits in a groundnut (Arachis bypogaea L.) mapping population. Field Crop Res. 103:187-197. 
Kasuga, M., Q, Liu, S, Miura, K. Yamaguchi-Shinozaki, and K. Shinozaki. 1999. Improving plant drought, salt, and freezing tolerance by gene transfer of a single stress inducible transcription factor. Nat. Biotechnol. 17:287-291

Kasuga, M., S. Miura, K. Shinozaki, and K. Yamaguchi-Shinozaki. 2004. A combination of the Arabidopsis DREB1A gene and stress-inducible $r d 29 A$ promoter improved drought- and low-temperature stress tolerance in tobacco by gene transfer. Plant Cell Physiol. 45:346-350

Katiyar-Agarwal, S., M. Agarwal, and A. Grover. 1999. Emerging trends in agricultural biotechnology research: use of abiotic stress induced promoter to drive expression of a stress resistance gene in the transgenic system leads to high level stress tolerance associated with minimal negative effects on growth. Curr. Sci. 77:1577-1579.

Kishor, P.B.K., Z. Hong, G.H. Miao, C.A.A. Hu, and D.P.S. Verma. 1995. Overexpression of $\Delta^{1}$-pyrroline-5-carboxylate synthetase increases proline production and confers osmotolerance in transgenic plants. Plant Physiol. 108:1387-1394.

Kodama, H., T. Hamada, G. Horiguchi, M. Nishimura, and K. Iba. 1994. Genetic enhancement of cold tolerance by expression of a gene for chloroplast $\omega-3$ fatty acid desaturase in transgenic tobacco. Plant Physiol. 105:601-605.

Lee, S.S., H.S. Cho, G.M. Yoon, J.W. Ahn, H.H. Kim, and H.S. Pai. 2003. Interaction of NtCDPK1 calcium-dependent protein kinase with NtRpn3 regulatory subunit of the 26S proteasome in Nicotiana tabacum. Plant J. 33:825-840.

McKersie, B.D., S.R. Bowley, E. Harjanto and O. Leprince, 1996.Water-deficit tolerance and field performance of transgenic alfalfa over-expressing superoxide dismutase. Plant Physiol. 111:1177-1181.

Morgan, J.M. 1984. Osmoregulation and water stress in higher plants. Annu. Rev. Plant Physiol. 35:299-348.

Nanjo, T., M. Kobayashi, Y. Yoshiba, Y. Kakubari, K.Yamaguchi-Shinozaki, K. Shinozaki. 1999. Antisense suppression of proline degradation improves tolerance to freezing and salinity in Arabidopsis thaliana. FEBS Lett. 461:205-210

Nigam, S.N., S. Chandra, K. Rupa Sridevi, M. Bhukta, A.G.S. Reddy, N.R. Rachaputi, G.C. Wright, et al. 2005. Efficiency of physiological trait-based and empirical selection approaches for drought tolerance in groundnut. Ann. Appl. Biol. 146: 433-439.

Pardo, J.M., M.P. Reddy, and S. Yang. 1998. Stress signaling through $\mathrm{Ca}^{2+} /$ Calmodulin dependent protein phosphatase calcineurin mediates salt adaptation in plants. Proc. Natl. Acad. Sci. 95:9681-9683.

Passioura, J.B. 1977. Grain yield, harvest index and water use of wheat. J. Aust. Inst. Agri. Sci. 43:21.

Pellegrineschi A, M. Reynolds, M. Pacheco, R. M. Brito, R. Almeraya, K. YamaguchiShinozaki, and D. Hoisington. 2004. Stress-induced expression in wheat of the Arabidopsis thaliana DREB1A gene delays water stress symptoms under greenhouse conditions. Genome 47:493-500

Pilon-Smits, E.A.H., M.J.M. Ebskamp, M.J.W. Jeuken, I.M. van der Meer, R.G.F. Visser, P.J. Weisbeek, and J.C.M. Smeekens. 1996. Microbial fructan production in transgenic potato plants and tubers. Ind. Crops Prod. 5:35-46

Pilon-Smits, E.A.H., M.J.M. Ebskamp, M.J. Paul, J.W. Jeuken, Weisbeek, and S.C.M. Smeekens. 1995. Improved performance of transgenic fructan-accumulating tobacco under drought stress. Plant Physiol. 107:125-130 
Pilon-Smits, E.A.H., N. Terry, T. Sears, and K. van Dun. 1999. Enhanced drought resistance in fructan-producing sugar beet. Plant Physiol. Biochem. 37:313-317

Ray, J.D., and T.R. Sinclair. 1997. Stomatal closure of maize hybrids in response to soil drying. Crop Science 37:803-807.

Ray, J.D., and T.R. Sinclair. 1998. The effect of pot size on growth and transpiration of maize and soybean during water deficit stress. J. Exp. Bot. 49(325): 1381-1386.

Ritchie, G.A. 1982. Carbohydrate reserves and root growth potential in Douglas fir seedlings before and after cold storage. Can.J. For. Res. 12:905-912.

Romero, C., J.M. Belles, J.L. Vaya, R. Serrano, and F.A. Culianez-Macia. 1997. Expression of the yeast trehalose-6-phosphate synthase gene in transgenic tobacco plants: pleiotropic phenotypes include drought tolerance. Planta 201: 293-297

Sakuma, Y., K. Maruyama, k. Osakabe, F. Qin, M. Seki, K. Shinozaki, and K. Yamaguchi-Shinozaki. 2006a. Functional analysis of an Arabidopsis transcription factor, DREB2A, involved in drought-responsive gene expression. Plant Cell 18:1292-1309

Serraj, R., L.C. Purcell, and T.R. Sinclair. 1999 Symbiotic $\mathrm{N}_{2}$ fixation response to drought. J. Exp. Bot. 50:143-155.

Serraj, R., and T.R. Sinclair. 2002. Osmolyte accumulation: Can it really help increase crop yield under drought conditions? Plant Cell Environ. 25:333-341

Sharma K.K., and M. Lavanya. 2002. Recent developments in transgenics for abiotic stress in legumes of the semi-arid tropics. In Genetic engineering of crop plants for abiotic stress, ed. M. Ivanaga M. 61-73, JIRCAS Working Report No. 23. Tsukuba, Japan: JIRCAS.

Sharma, K.K., and R. Ortiz. 2000. Program for the application of the genetic engineering for crop improvement in the semi-arid tropics. In Vitro Cell. Develop. Biol. (Plant) 36:83-92.

Sheshshayee, M.S., H. Bindumadhava,, N.R. Rachaputi,, T.G. Prasad,, M. Udayakumar, and G.C. Wright. 2006. Leaf chlorophyll concentration relates to transpiration efficiency in peanut. Oxford: Blackwell.

Shinwari, Z.K. 1999. Function and regulation of genes that are induced by dehydration stress. Biosci. Agric. 5:39-47.

Shinwari, Z.K., K. Nakashima, S.Miura, M. Kasuga, M. Seki, K. Yamaguchi-Shinozaki, and K. Shinozaki. 1998. An Arabidopsis gene family encoding DRE/CRT binding proteins involved in low-temperature-responsive gene expression. Biochem. Biophys. Res. Commun. 50:161-170

Sinclair, T.R., and M.M. Ludlow. 1986. Influence of soil water supply on the plant water balance of four tropical grain legumes. Aust. J. Plant Physiol. 13:329-341

Sinclair, T.R., L.C. Purcell, and C.H. Sneller. 2004. Crop transformation and challenge to increase yield potential. Trends Plant Sci. 9:70-75

Sivamani, E., A. Bahieldin, J.M. Wraith, T. Al-Niemi, W.E. Dyer, T.H.D. Ho, and R. Qu. 2000. Improved biomass productivity and water use efficiency under water deficit conditions in transgenic wheat constitutively expressing the barley HVA1 gene. Plant Sci. 155:1-9.

Slafer, G.A. 2003. Genetic basis of yield as viewed from a crop physiologist's perspective. Ann. Appl. Biol. 142(2): 117-128.

Snape, J.W., K. Butterworth, E. Whitechurch, and A.J. Worland. 2001. Waiting for fine times: Genetics of flowering time in wheat. Euphytica 119:185-190. 
Sun, W., C. Bernard, B. van de Cotte, M.V. Montagu, and N. Verbruggen. 2001. At-HSP17.6A, encoding a small heat-shock protein in Arabidopsis, can enhance osmotolerance upon overexpression. Plant J. 27:407-415.

Tarczynski, M. C., R.G. Jensen, and H.J. Bohnert. 1993. Stress protection of transgenic tobacco by production of the osmolyte mannitol. Science259: 508-510.

Turner, N.C., A. Shahal, J.D. Berger, S.K. Chaturvedi, R.J. French, C. Ludwig, D.M. Mannur, S.S. Singh, and H.S. Yadava 2007. Osmotic adjustment in chickpea (Cicer arietinum L.) results in no yeild benefit under terminal drought. J. Exp. Bot. 58: 187-194.

Turner, N.C., and M.M. Jones. 1980. Turgor maintenance by osmotic adjustment: a review and evaluation. In Adaptation of plants to water and high temperature stress, eds. N.C. Turner, and P.J. Kramer, pp. 84-104. New York: Wiley Intersience

Umezawa, T., M. Fujita, Y. Fujita, K. Yamaguchi-Shinozaki, and K. Shinozaki. 2006. Engineering drought tolerance in plants: discovering and tailoring genes to unlock the future. Curr. Opin. Biotechnol. 17:113-122.

Vadez, V., S. Rao, J. Kholova, L. Krishnamurthy, J. Kashiwagi, P. Ratnakumar, K.K. Sharma, P. Bhatnagar-Mathur, and P.S. Basu. 2008. Roots research for legume tolerance to drought: Quo vadis? J. Food Legumes 21(2): 77-85.

Vadez, V., S. Rao, K.K. Sharma, P. Bhatnagar-Mathur and M.J. Devi. 2007. DREB1A allows for more water uptake by a large modification in the root/shoot ratio under water deficit. Int. Ara. Newsletter. 27:27-31.

Wang, W. B. Vinocur, and A. Altman. 2003. Plant responses to drought, salinity and extreme temperatures: towards genetic engineering for stress tolerance. Planta 218:1-14.

Wenzel, and Wayne. March 14, 2008. H2O Optimizers, Farm Journal.

Wright, G.C., K.T. Hubick, G.D. Farquhar, and R.C.N. Rao. 1993. Genetic and environmental variation in transpiration efficiency and its correlation with carbon isotope discrimination and specific leaf area in peanut. In Stable isotopes and plant carbon/water relations, eds. J.R. Ehleringer, A.E. Hall, and G.D. Farquhar, pp. 247-267. San Diego, CA: Academic Press.

Wright, G.C., R.C. Nageswara Rao, and G.D. Farquhar. 1994. Water-use efficiency and carbon isotope discrimination in peanut under water deficit conditions. Crop Sci. 34:92-97.

Xu, D., X. Duan, B. Wang, B. Hong, T.H.D. Ho, and R. Wu. 1996. Expression of a late embryogenesis abundant protein gene, HVA1, from barley confers tolerance to water deficit and salt stress in transgenic rice. Plant Physiol. 110: 249-257.

Yamada, M., H. Morishita, K.Urano, N. Shiozaki, K. Yamaguchi-Shinozaki, K. Shinozaki and Y. Yoshiba. 2005. Effects of free proline accumulation in petunias under drought stress. J Exp. Bot. 56:1975-1981.

Zhang, H., Z. Huang, B. Xie, Q.Chen, X. Tian, X. Zhang, H.B. Zhang, X. Lu, D. Huang, and R. Huang. 2004. Ethylene, jasmonate, abscisic acid and $\mathrm{NaCl}$ responsive tomato transcription factor JERF1 modulates expression of GCC box-containing genes and salt tolerance in tobacco. Planta 220:262-270.

Zhu, B., J. Su, M. Chang, D.P.S. Verma, Y.L. Fan and R. Wu. 1998. Overexpression of delta1-pyrroline-5-carboxylate synthase gene and analysis of tolerance to water and salt stress in transgenic rice. Plant Sci. 199:41-48. 